\title{
DESARROLLO E IMPLEMENTACIÓN DE UN SISTEMA DE CONTROL EN UNA PLANTA PILOTO HÍBRIDA
}

\author{
Maria P. Marcos, César de Prada \& José Luis Pitarch \\ Dpto. Ingeniería de Sistemas y Automática, Escuela de Ingenierías Industriales, Universidad de Valladolid. \\ C/ Real de Burgos s/n, 47011, Valladolid, \{maria.marcos | prada|jose.pitarch\}@autom.uva.es
}

\begin{abstract}
Resumen
En este trabajo se describe el desarrollo e implementación de un sistema de control de una planta piloto química. Dicha planta piloto tiene la característica de que el componente principal del proceso, la reacción exotérmica, está siendo simulado. Antes de configurar el sistema SCADA, se ha modelado y simulado el proceso, y definido el sistema de control que se llevará a cabo. Destacar que el sistema SCADA además de proporcionar una interfaz gráfica de visualización del sistema, conecta la tarjeta de adquisición de datos de la planta con diferentes módulos externos accesibles como servidores OPC con diferentes funcionalidades: cuatro capaces de realizar cálculos PID y que de forma conjunta constituyen el sistema de control, y uno que funciona como un bloque calculador.
\end{abstract}

Palabras clave: Planta híbrida, reacción simulada, EcosimPro, SCADA Intouch, S-IMC.

\section{MOTIVACIÓN Y OBJETIVO}

En los últimos años se han desarrollado novedosas técnicas basadas en el modelado de procesos con el fin de mejorar la industria de procesos. Sin embargo, la validez de dichas técnicas debe ser corroborada experimentalmente en una planta real. No obstante, la experimentación es una técnica cara que además puede suponer complicaciones añadidas con respecto a la seguridad y al impacto ambiental, por ello se ha decidido llevar a cabo una opción intermedia, construyendo una planta piloto híbrida, es decir, una planta que mantiene su estructura e instrumentación y opera a tiempo real pero donde parte del proceso está siendo simulado.

Esta opción es una alternativa a los laboratorios virtuales [1] [2] los cuales están basados en simulaciones, puesto que, aunque la simulación es una técnica muy avanzada actualmente, la realidad de los procesos siempre presenta inconvenientes adicionales con los que lidiar. Esto se debe a que los modelos empleados en simulación son una aproximación más o menos precisa de la realidad y, por tanto, los resultados derivados de las simulaciones pueden diferir bastante de la realidad experimental.
El interés de proponer una planta piloto híbrida es que se pueda experimentar con ella sin incurrir en los costosos gastos de instrumentación para la medida de concentración de compuestos químicos, adquisición de materias primas, la problemática del tratamiento de residuos, etc., es decir, se obtiene una solución más favorable económica y ecológicamente. Aunque esta forma de operar tampoco reproduce totalmente el comportamiento real del sistema, sí que puede aproximar de una manera muy fiel la realidad además de permitir la experimentación en un rango de operación más amplio.

Un caso conocido es la aplicación de plantas piloto híbridas por la universidad de Santamaría de Valparaíso, Chile en el campo de las columnas de flotación [3]. En la minería, las columnas de flotación son uno de los equipos más utilizados para obtención de minerales valiosos procedentes de la ganga, pero realizar experimentos con estos equipos es costoso debido a sus condiciones de operación. La implementación del sistema híbrido viene dado por la sustitución de la alimentación por agua y la consiguiente simulación de concentraciones de los componentes tanto a la entrada como a la salida.

La finalidad de la planta piloto es que tanto los alumnos como los investigadores de la Universidad de Valladolid puedan experimentar diversas estrategias de control y optimización, modificando la dificultad de las reacciones a simular.

Así pues, este trabajo tiene como objeto configurar el sistema de control y supervisión de una planta piloto química, en la que el componente principal del proceso, es decir, la reacción exotérmica, va a ser simulada con el fin de que se pueda experimentar con ella, actuar sobre el control de las diferentes variables del proceso y observar su dinámica. Para llevar a cabo esta configuración primero se ha modelar el proceso y diseñar y simular el sistema de control que se va implementar.

El resto del trabajo se organiza como sigue. La siguiente sección describe la planta piloto, los diferentes equipos y la instrumentación que la forman, y se comentan las consideraciones que se han de tener en cuenta al realizar un montaje híbrido. En la Sección 3 
se muestra el modelo obtenido del proceso y la simulación de dicho modelo para las condiciones del montaje experimental. La Sección 4 se divide en el diseño del sistema de control según los resultados de la sección anterior, y la implementación de dicho sistema mediante un SCADA ("Supervisory Control And Data Acquisition", supervisión, control y adquisición de datos). Por último, la Sección 5 expone las conclusiones generales a las que se ha llegado junto con las líneas futuras.

\section{DESCRIPCIÓN DE LA PLANTA}

El elemento principal de la planta piloto es un reactor continuo de tanque agitado (RCTA) con una camisa refrigerante etiquetado como R-101en la Figura 1, en el que se quiere llevar a cabo la simulación de la reacción un mol de reactivo A para obtener un mol de producto B.

Una reacción química conlleva altos costes de materia prima, tratamiento de productos, problemas de seguridad e impacto ambiental, etc., por ello se ha decido que la parte experimental de la reacción sea sustituida por un cálculo en simulación cuyos resultados puedan visualizarse en forma de concentración y aplicar el calor de reacción que se estaría produciendo mediante una resistencia eléctrica variable, cuya potencia corresponda a la cantidad de calor de reacción calculado. Así pues, para simular dicho calor, parte del fluido del interior del reactor se lleva a un tanque (T-102) en el que hay una resistencia calefactora que aumenta la temperatura del fluido, devolviéndose nuevamente al reactor.

Para poder simplificar el comportamiento real del sistema se tienen que tener en cuenta un par de consideraciones:
En primer lugar, para que la hidrodinámica del proceso quede bien representada, las propiedades físicas de los diferentes fluidos del proceso real (densidad, viscosidad, capacidad calorífica, etc. de cada corriente) han de ser prácticamente invariantes con las propiedades que si varían a lo largo del proceso (temperatura y concentración) y así poder sustituir los reactivos y productos por un fluido más manejable, en este caso el agua.

En segundo lugar, puesto que parte del proceso se está simulando, es necesario disponer de modelos matemáticos que permitan calcular de forma precisa las variables que no se pueden medir (debido a que la reacción se está simulando) según las condiciones de operación establecidas.

Consecuentemente, si se dispone de un modelo y la planta piloto puede ser operada utilizando estos fluidos alternativos, se puede desarrollar un sistema híbrido con el que poder experimentar parcialmente. El comportamiento real queda simplificado, pero las características hidrodinámicas y térmicas se mantienen fieles a la realidad. Además, empleando un modelo adecuado, las concentraciones finales del proceso pueden ser calculadas razonablemente (no medidas) en un amplio rango de operación.

Además del reactor y del tanque calefactor, la planta cuenta con diversos equipos auxiliares; tanque de almacenamiento de reactivos (T-101), bomba peristáltica de impulsión de reactivos al reactor (P-101), bomba de diafragma a la salida del reactor (P-102), bomba peristáltica de cabezal doble para extraer $(\mathrm{P}$ 103A) e introducir (P-103B) el líquido que se lleva al tanque calefactor, válvula de diafragma que controla el fluido refrigerante (V-101), y unidad de potencia trifásica a tiristores que proporciona la potencia a la resistencia calefactora (J-101).

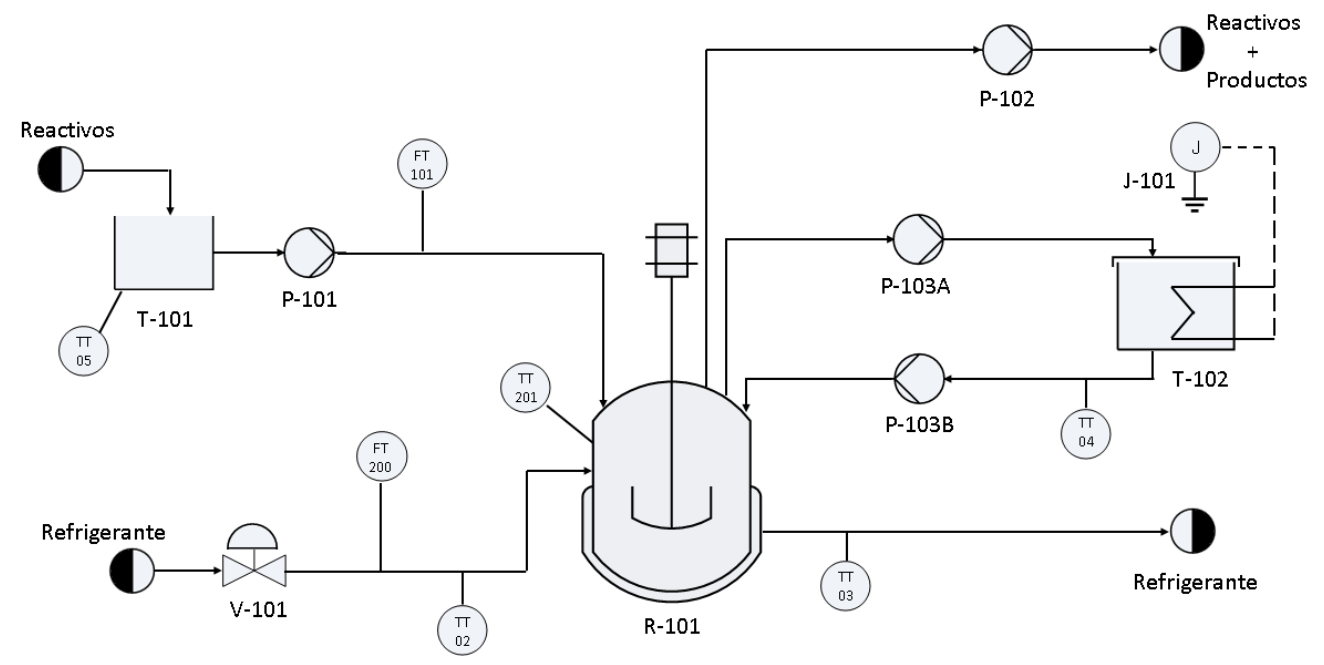

Figura 1 Diagrama del proceso 
Como instrumentación para poder implementar el sistema de control se cuenta con: dos caudalímetros electromagnéticos para medir los caudales de alimento al reactor (FT101) y a la camisa refrigerante (FT200) y 5 termorresistencias con su correspondiente transmisor para medir la temperatura en: el tanque de alimentación (TT05), dentro del reactor (TT201), a la entrada (TT02) y salida (TT03) de la camisa y en el tanque calefactor (TT04), todo ello conectado a una tarjeta de adquisición de datos (DAQ) para poder controlar el sistema mediante el ordenador.

\section{MODELADO Y SIMULACIÓN}

En primer lugar, se ha desarrollado un modelo matemático del sistema que refleja con precisión las características más importantes del mismo, con el objetivo de simular posteriormente el comportamiento y diseñar el sistema de control.

Para llevar a cabo este modelado se han aplicado los principios de conservación de masa y energía y otras leyes particulares del dominio de aplicación. Además, se ha considerado que en el interior hay un comportamiento de mezcla perfecta, es decir se ha descartado que haya caminos de flujo preferentes o zonas muertas, y se ha considerado que el caudal de entrada es el mismo que el de salida, tanto en el caso del reactor como en la camisa refrigerante.

El modelo obtenido es:

$$
\begin{gathered}
\frac{d C_{A}}{d t}=\frac{Q_{v}}{V}\left(C_{A 0}-C_{A}\right)-r_{A} \\
V \rho C_{p} \frac{d T}{d t}=Q_{v}\left(T_{i}-T\right)-Q_{i n t}+Q_{\text {reac }} \\
V_{j} \rho_{j} C_{p j} \frac{d T_{j}}{d t}=Q_{v j}\left(T_{j i}-T_{j}\right)+Q_{i n t} \\
Q_{\text {reac }}=\Delta H V r_{A} \\
Q_{\text {int }}=U A\left(T-T_{j}\right) \\
r_{A}=K_{0} e^{\left(\frac{-E a}{R T}\right)} C_{A} \\
X=1-\frac{C_{A}}{C_{A 0}}
\end{gathered}
$$

Donde:

- A: es el área de intercambio de calor entre el reactor y la camisa, en $\mathrm{m}^{2} .\left(0.1485 \mathrm{~m}^{2}\right)$

- $C_{A}$ : es la concentración del compuesto A en el reactor, en $\mathrm{mol} / \mathrm{L}$.
- $C_{A 0}$ : es la concentración del compuesto A inicial, en $\mathrm{mol} / \mathrm{L}$. (5 $\mathrm{mol} / \mathrm{L})$.

- Cp: calor especifico del fluido, en $\mathrm{kJ} / \mathrm{kg} \cdot \mathrm{K},(4.18$ $\mathrm{kJ} / \mathrm{kg} \cdot \mathrm{K})$.

- $C p_{j}$ : calor especifico del refrigerante, en $\mathrm{kJ} / \mathrm{kg} \cdot \mathrm{K}$, $(4.18 \mathrm{~kJ} / \mathrm{kg} \cdot \mathrm{K})$.

- $E_{a}$ : es la energía de activación de la reacción, en $\mathrm{J} / \mathrm{mol}$. (2420 J/mol)

- $\Delta H$ : variación de entalpía de reacción molar, en $\mathrm{kJ} / \mathrm{mol}(20 \mathrm{~kJ} / \mathrm{mol})$.

- $K_{0}$ : es el factor preexponencial o factor de frecuencia, en $\min ^{-1}$ (1.38 min-1)

- $Q_{\text {int }}$ : es el calor intercambiado entre el reactor y la camisa refrigerante, en $\mathrm{kJ} / \mathrm{min}$.

- $Q_{\text {reac }}$ : es el calor de reacción simulado mediante el amplificador de potencia, en $\mathrm{kJ} / \mathrm{min}$.

- $Q_{v}$ : es el caudal de reactivos, en $\mathrm{L} / \mathrm{min}$.

- $Q_{v j}$ : es el caudal de refrigerante, en $\mathrm{L} / \mathrm{min}$.

- $R$ : es la constante universal de los gases ideales, en $\mathrm{J} / \mathrm{mol} \cdot \mathrm{K} .(8.31 \mathrm{~J} / \mathrm{mol} \cdot \mathrm{K})$

- $r_{A}$ : es la velocidad de reacción, en $\mathrm{mol} / \mathrm{L} \cdot \mathrm{min}$.

- $T$ : es la temperatura a la salida del reactor, en ${ }^{\circ} \mathrm{C}$.

- $T_{i}$ : es la temperatura a la entrada del reactor, en ${ }^{\circ} \mathrm{C} .\left(15^{\circ} \mathrm{C}\right)$

- $T_{j}$ : es la temperatura de la camisa refrigerante a la salida, en ${ }^{\circ} \mathrm{C}$.

- $T_{j i}$ : es el temperatura de la camisa refrigerante a la entrada, en ${ }^{\circ} \mathrm{C} .\left(10^{\circ} \mathrm{C}\right)$

- $U$ : es el coeficiente global de transmisión de calor, en $\mathrm{kJ} / \mathrm{min} \cdot \mathrm{m}^{2} \cdot \mathrm{K}\left(202,64 \mathrm{~kJ} / \mathrm{min} \cdot \mathrm{m}^{2} \cdot \mathrm{K}\right)$.

- $V$ : es el volumen del reactor, en L. $(4.67 \mathrm{~L})$

- $V_{j}$ : es el volumen de la camisa, en L. $(1 \mathrm{~L})$

- $X$ : es la conversión de la reacción ( $\mathrm{s} / \mathrm{u})$.

- $\rho$ : densidad del fluido, en $\mathrm{kg} / \mathrm{L} .(1 \mathrm{~kg} / \mathrm{L})$

- $\rho_{j}$ : densidad del refrigerante, en $\mathrm{kg} / \mathrm{L} .(1 \mathrm{~kg} / \mathrm{L})$

De las diferentes variables que describen el proceso, algunas de ellas son constantes que dependen bien del montaje experimental o de la reacción a simular, y otras son variables de estado del proceso. Por tanto, se puede simular el comportamiento del sistema para un montaje experimental concreto y una reacción determinada.

Para el montaje experimental llevado a cabo se ha realizado una simulación del proceso mediante el entorno de simulación EcosimPro [4] modificando los caudales de alimentación al reactor y de refrigerante. El resultado de dicha simulación puede observarse en la Figura 2 .

Los valores de los parámetros que se han utilizado para la simulación (entre paréntesis en la descripción de las abreviaturas) corresponden a los datos del montaje y las temperaturas de entrada a las de la toma de agua de red en Valladolid en marzo. 

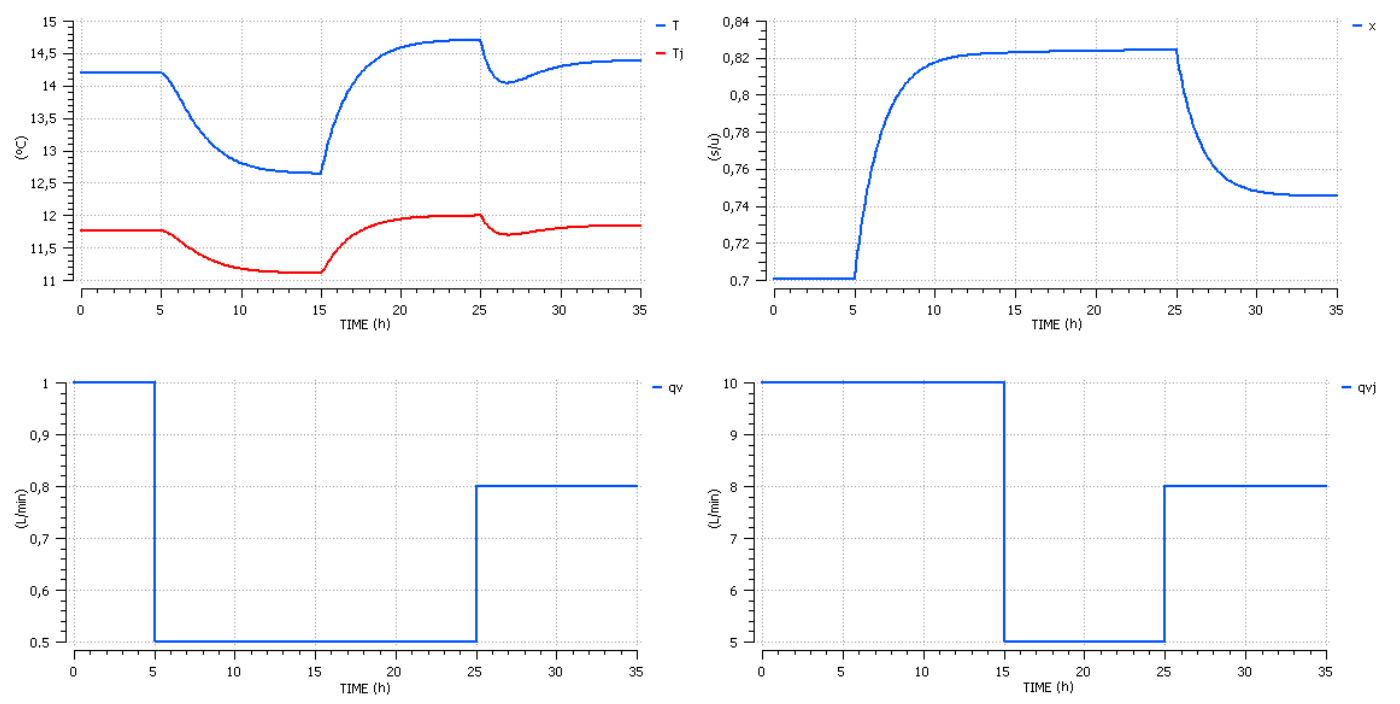

Figura 2. Respuesta dinámica del sistema a) Temperaturas b) Conversión

c) Caudal de refrigerante d) Caudal de alimentación

Partiendo del estado estacionario, se han introducido saltos en el caudal de alimentación a los 5 y 25 minutos (Figura 2.d) y en el caudal de refrigerante a los 15 y 25 minutos (Figura 2.c). Si se analiza la evolución del sistema hasta el equilibrio para las variables más importantes del sistema (conversión y temperatura) se observa que la conversión únicamente se ve afectada positivamente ante una disminución del caudal de alimentación (Figura 2.b), mientras que la temperatura tanto del reactor como de la camisa depende de ambos caudales, pero con dinámicas opuestas: una disminución del caudal de alimentación disminuye las temperaturas (ganancia positiva) mientras que una disminución del caudal de refrigerante las aumenta (ganancia negativa).

Cabe destacar que la ganancia de la temperatura respecto al caudal de alimentación es mayor que la ganancia respecto al caudal de refrigerantes puesto que, como se aprecia en la Figura 2.a, ante un cambio de ambos caudales, las temperaturas se ven más afectadas por el cambio del caudal de alimentación, pese a ser un cambio en menor proporción.

\section{SISTEMA DE CONTROL}

Existen diferentes estrategias de control que se puede realizar para un mismo proceso según las necesidades que se requieran. En este caso solo se van a establecer dos lazos simples de control, uno para controlar la conversión y otro para controlar la temperatura del reactor. En las siguientes subsecciones se explicará respectivamente, como se ha realizado el diseño de estos controladores y como se ha conectado el sistema de control a la planta real mediante un sistema HMI.

\subsection{DISEÑO DEL CONTROLADOR}

En una reacción las dos variables más importantes a controlar son la temperatura que se alcanza en el interior del reactor, sobre todo cuando dicha reacción es exotérmica, y la concentración de producto o conversión de la reacción. Para llevar a cabo este control, las variables manipuladas son el caudal de alimentación al reactor, y el caudal de refrigerante.

A partir del modelo obtenido se han realizado diferentes simulaciones manipulando las entradas del sistema (los diferentes caudales) con el fin de obtener la matriz de ganancias relativas (RGA) y así saber la relación entre las variables manipuladas y las controladas, y poder realizar la sintonía teórica de los controladores PID que controlen el sistema.

Mediante los datos de simulación de los incrementos de las salidas respectos a los saltos introducidos en las entradas se puede calcular una aproximación las ganancias estacionarias de cada par y por tanto la matriz de ganancias estacionarias:

$$
\left[\begin{array}{l}
T \\
x
\end{array}\right]=\left[\begin{array}{cc}
-0.0842 & 0.0651 \\
-1.81 \cdot 10^{-3} & -0.246
\end{array}\right]\left[\begin{array}{c}
Q_{v j} \\
Q_{v}
\end{array}\right]
$$

Una vez obtenida dicha matriz, para obtener la RGA se han de calcular las diferentes ganancias relativas del sistema [5], donde la ganancia relativa de cada par entrada - salida se calcula a partir de las ganancias estacionarias en los casos extremos de cada lazo, es decir, cuando el resto de lazos del sistema están abiertos y cuando el resto de lazos del sistema están con control perfecto. Sin embargo, puesto que puede demostrarse [5] que, para un sistema de dos entradas con dos salidas, la ganancia estacionaria cuando el resto de lazos 
del sistema están controlados depende del resto de ganancias cuando el resto de lazos están abiertos, a partir de los resultados de la matriz de ganancias estacionarias (8), la matriz de ganancias relativas queda tal que:

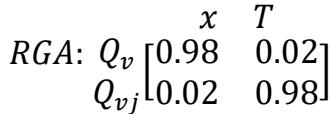

A la vista de los resultados obtenidos, se establecen dos lazos de control con el siguiente emparejamiento: el lazo 1 que controlará la conversión a partir del caudal de alimentación (Figura 3 ) y el lazo 2 que controlará la temperatura del reactor a partir del caudal de refrigerante (Figura 4). Además, puesto que los valores de la diagonal son aproximadamente 1 se puede deducir que la interacción entre ambos lazos de control será prácticamente nula.
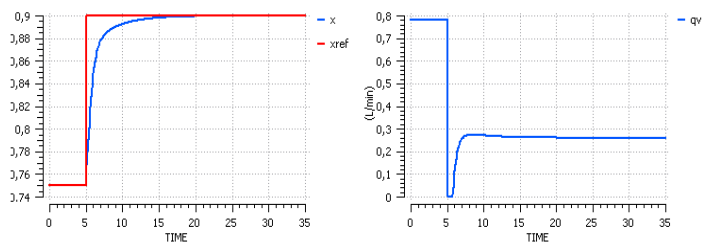

Figura 3. a) SP y PV-Conversión del reactor b) CV - Caudal de alimentación

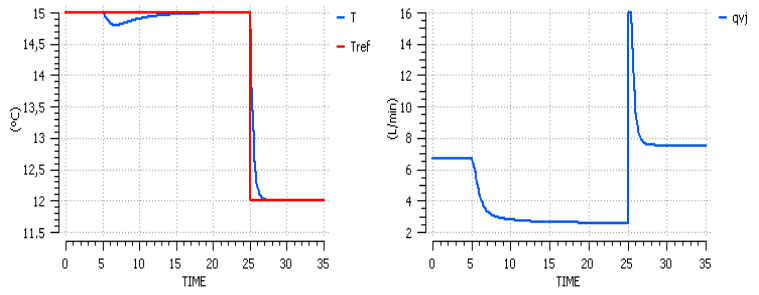

Figura 4. a) SP y PV - Temperatura del reactor b) $\mathrm{CV}$ - Caudal de refrigerante

Se ha de señalar que para realizar la sintonía de los controladores se ha utilizado el método de S-IMC [6] en lazo abierto, tomando $\lambda$ igual a 2 , para tener un control ni demasiado conservador ni demasiado agresivo.

A partir de la Figura 3 y la Figura 4 se puede apreciar que al modificar los estados de referencia, el sistema responde rápidamente para llegar al nuevo punto de consigna. También cabe destacar que, tal y como se esperaba, hay poco acoplamiento entre lazos pues la temperatura apenas varía ante un cambio de caudal de alimentación y la conversión no varía ante cambios en el caudal de refrigerante.

\subsection{IMPLEMENTACIÓN}

Una vez diseñado el sistema de control de la planta, se procede a implementarlo, teniendo en cuenta que el caudal de alimentación se controlará con la velocidad proporcionada a la bomba de alimentación (P-101) y el caudal de refrigerante se regulará mediante la válvula V-101. Como no se manipulan directamente los caudales pero sí son medidos, el sistema de control será parecido al obtenido teóricamente pero añadiendo en ambos casos un lazo interno con el que controlar los correspondientes actuadores, es decir, la bomba P-101 y la válvula V-101. Así pues, se establecerán dos lazos en cascada, en el primero el lazo externo controlará la conversión a partir del caudal de alimentación de referencia y el lazo interno comparará dicho caudal de referencia con el caudal real y modificará la velocidad de la bomba. Por otra parte, el segundo lazo en cascada hará lo mismo con la temperatura, el caudal de refrigerante y la válvula respectivamente (Véase Figura 5).

Actualmente mediante el software Intouch de la empresa Wonderware [7] se ha diseñado un sistema SCADA que, por un lado conecta la planta (mediante la tarjeta DAQ) con el sistema de control, y por otro proporciona una interfaz gráfica con la que supervisar y controlar la planta. (Véase Figura 6)

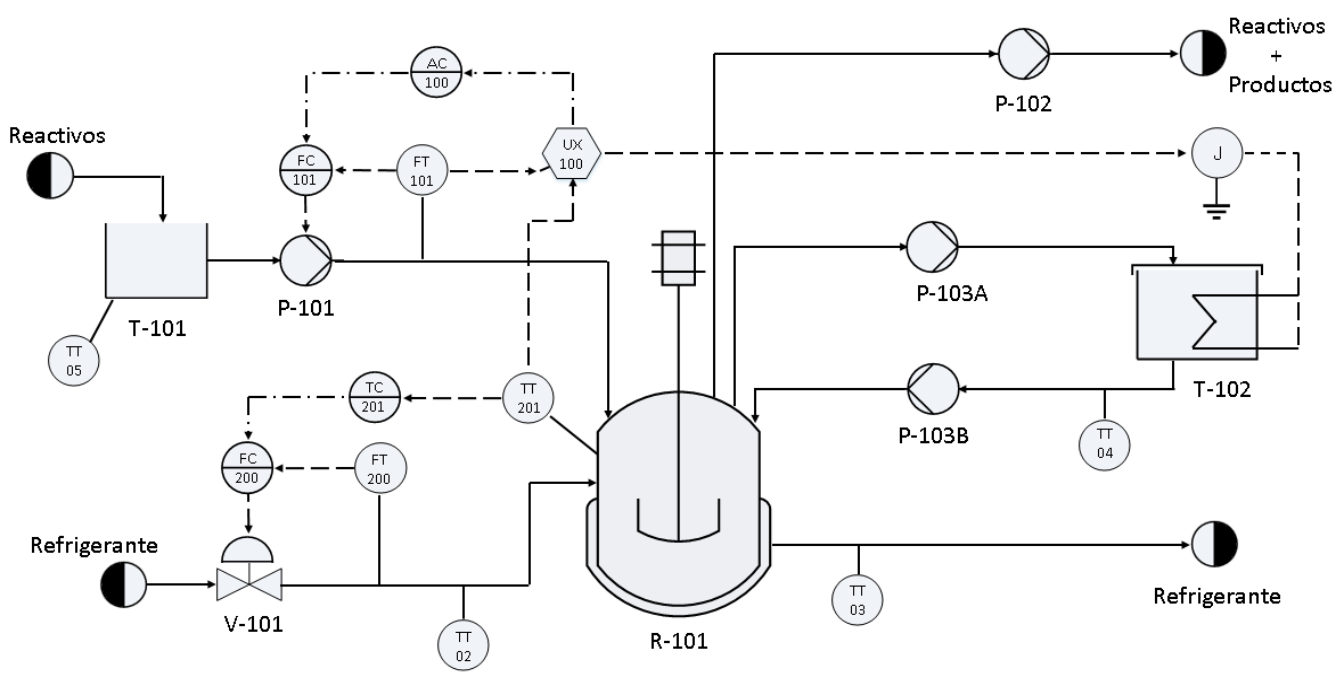

Figura 5 . Diagrama P\&I del proceso 


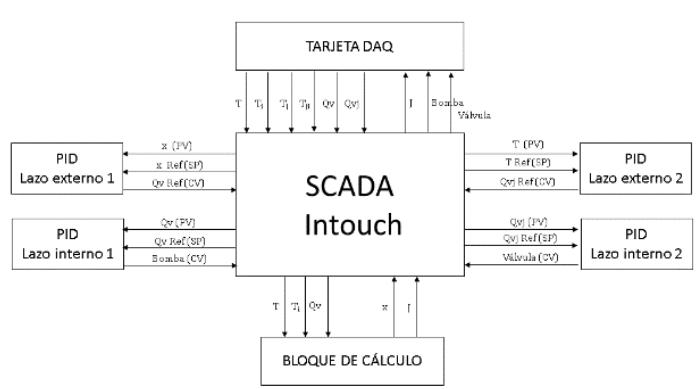

Figura 6. Integración de los diferentes subsistemas

Mediante la tarjeta DAQ se obtienen los valores medidos de las temperaturas tanto del reactor, a la entrada y la salida de la camisa refrigerante, y del tanque de almacenamiento de reactivos, como de los caudales de alimentación y de refrigerante, además de enviar las señales de control a la bomba de alimentación y a la válvula de refrigerante, y la señal correspondiente a la unidad de potencia para que suministre el calor de reacción adecuado.

Por otra parte, el sistema de control se ha implementado utilizando 4 módulos externos accesibles como servidores OPC. Cada uno de estos módulos, desarrollado mediante EcosimPro, actúa como un controlador PID que, tras suministrarle el valor en tiempo real de la variable del proceso que se quiere controlar, el punto de consigna y los parámetros del regulador, calcula la correspondiente variable manipulada [8].

Cabe destacar que para que la planta reproduzca el comportamiento real del sistema, se ha tenido que desarrollar un quinto modulo externo que calcula la conversión y el calor de reacción que se estaría produciendo (y que ha de suministrar la unidad de potencia J-101 para simularlo) a partir de las variables medidas. Es decir mediante EcosimPro, se ha desarrollado un nuevo servidor OPC que actúa como bloque calculador, y en tiempo real, recibe el valor del caudal de alimento que se está midiendo y de la temperatura del reactor, resuelve las ecuaciones (1), (4), (6) y (7), y devuelva cómo salida la conversión y el calor de reacción. A partir del valor de calor de reacción que devuelve el bloque calculador, el sistema SCADA envía la señal correspondiente a la unidad de potencia para suministrar dicho calor a la resistencia calefactora. En el diagrama P\&I de la Figura 5 se ha representado este bloque calculador con símbolo de ordenador identificado como UX100.

Por último, la Figura 7 muestra la pantalla principal de la interfaz gráfica del sistema SCADA que se ha diseñado, en la que se observan los valores en tiempo real de las diferentes variables del proceso. Además, esta pantalla permite encender y apagar la planta, establecer la forma de controlar los diversos lazos de control: forma manual (se ha de establecer el valor deseado de consigna) o automáticamente (se indica directamente la señal de control).

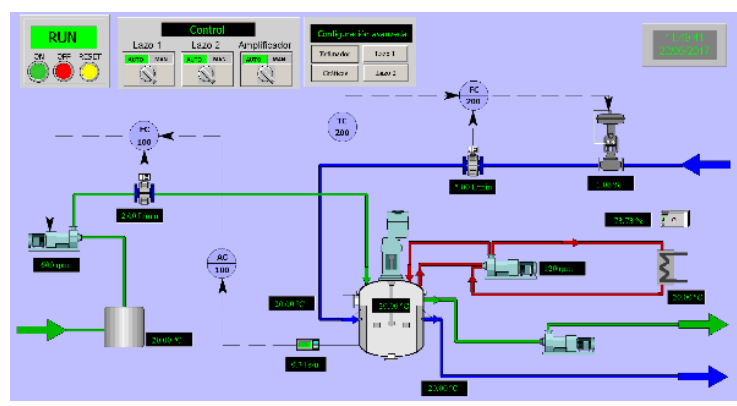

Figura 7. Pantalla principal del HMI

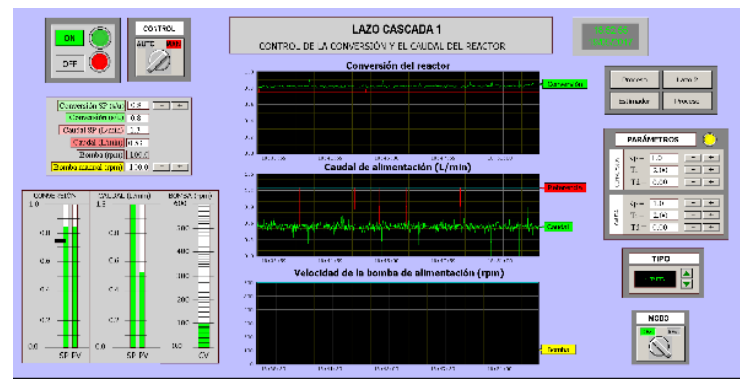

Figura 8. Pantalla de configuración del lazo 1

Además de esta pantalla, se han diseñado otras ventanas donde manipular las características más específicas del sistema de control. Un ejemplo de ello es la Figura 8, donde se muestra la pantalla de la configuración del Lazo 1 de control. Esta pantalla además de permitir visualizar las diferentes variables involucradas en el lazo de control de forma gráfica en tiempo real, muestra los valores numéricamente y en forma de diagrama de barras. También permite, nuevamente, establecer si se quiere utilizar un control manual o automático, elegir el modo de operación de los controladores (ideal o interactivo) y seleccionar el tipo de regulador (PID, PI-D, I-PD o I). Por último, la característica más importante de esta pantalla es que habilita la posibilidad de cambiar la sintonía de los parámetros de cada controlador. Así mismo, se ha diseñado otra pantalla equivalente para la visualización de las características del Lazo 2.

Por último, se han desarrollado otras dos pantallas de configuración avanzada, una para ver gráficas históricas de las diferentes variables involucradas en el proceso, y una que permite especificar los parámetros propios de la reacción que se quiere simular $\left(E_{a}, \Delta H\right.$, $k_{0}$, etc.).

\section{CONCLUSIONES}

Con el trabajo realizado hasta ahora se ha obtenido un buen modelo matemático que simula el comportamiento de la planta piloto que se quiere controlar y se ha diseñado un sistema de control con desacoplamiento estático que permite controlar las variables más importantes del proceso, a ser la conversión de la 
reacción y la temperatura del interior del reactor, teniendo poca interacción entre ambos lazos.

Una vez se ajuste la sintonía de los controladores del sistema, se podrá utilizar la planta para simular reacciones de diferente complejidad según se establezcan los parámetros correspondientes desde el sistema SCADA Intouch. Además, desde la interfaz gráfica del sistema SCADA se podrá visualizar la dinámica del proceso y manipular la forma de operar del sistema de control. Es decir, el alumno o investigador que trabaje con la planta podrá experimentar el comportamiento de diferentes reacciones y probar diferentes metodologías de control de forma sencilla manipulando los diferentes parámetros de control desde la interfaz gráfica del sistema SCADA.

Como trabajo futuro se desea implementar un nuevo módulo externo accesible como servidor OPC que realice una optimización del sistema en tiempo real para ajustar las consignas de temperatura y conversión a las condiciones de cada instante, según una función objetivo determinada. Es decir, el plan de futuro es implementar una capa superior de optimización con la que comandar el sistema de control para lograr una operación óptima de la planta.

\section{Agradecimientos}

Este trabajo ha sido financiado parcialmente por la Unión Europea y el Gobierno de España con fondos MINECO/FEDER (DPI2015-70975-P).

\section{Referencias}

[1] J. M. Andújar y T. J. Mateo, «Diseño de Laboratorios Virtuales y/o Remotos. Un caso Práctico,» Revista Iberoamericana de
Automática e Informática Industrial RIAI, vol. 7 , no 1, pp. 64-72, 2010.

[2] R. Pastor, J. Sánchez y S. Dormido, «Web-based virtual lab and remote experimentation using easy java simulations,» IFAC Proceedings Volumes, vol. 38, no 1, pp. 103-108, 2005.

[3] L. G. Bergh, «A Hybrid Approach to Empirically Test Process Monitoring, Diagnosis and Control Strategies.,» Thaung K. (eds) Advanced Information Technology in Education. Advances in Intelligent and Soft Computing, vol. 126., 2012.

[4] EcosimPro, «EcosimPro,» 2016. [En línea]. Available: http://www.ecosimpro.com/.

[5] S. Skogestad y I. Postlehwaite, «3.4 Relative Gain Array (RGA),» de Multivariable Feedback Control, $2^{\mathrm{a}}$ ed., New York, John Wiley \& Sons, 2005, pp. 82-91.

[6] S. Skogestad, «Simple analytic rules for model reduction and PID,» Journal of Process Control, vol. 13, pp. 291-309, 2003.

[7] Wonderware, «Wonderware Spain,» 2016. [En línea]. Available: http://www.wonderware.es/.

[8] J. M. Zamarreño, M. J. de la Fuente y L. F. Acebes, «Desarrollo de un control PID accesible como servidor OPC,» de Jornadas de Automática, Bilbao, 2015. 